\title{
Distribución, Incidencia y Alternativas de Control de Rhizoctonia solani en el Cultivo Papa en el Estado Mérida, Venezuela
}

\author{
Rosaima García, Alba García, Camilo Garnica ${ }^{1}$
}

\begin{abstract}
Resumen
Con el objetivo de determinar el nivel de distribución e incidencia de la rhizoctoniasis de la papa (Rhizoctonia solani) en el estado Mérida, y buscar alternativas de manejo, se realizó un diagnóstico de campo en cinco municipios paperos y un estudio en la semilla informal ubicado en los silos de Pico El Águila proveniente de siete municipios productores; posteriormente se evaluaron bajo condiciones de invernaderos y campo alternativas de control con opción a ser incorporadas dentro de un programa de manejo de la enfermedad. En invernadero se evaluó el efecto sobre la enfermedad de los fungicidas Carboxin + Thiram, Mancozeb y Pencycuron (dosis media comercial) y dos biopreparados a base de aislamientos nativos de Trichoderma harzianum (200 g del producto a concentración de $2 \times 10^{11}$ UFC /200 L de agua) sobre semilla y suelo contaminado, bajo un diseño en bloques al azar de arreglo en factorial, con tres repeticiones. Mientras que en el campo experimental Mucuchíes (3100 msnm) se evaluaron los tratamientos: Pencycuron a dosis de $600 \mathrm{~g}$ del producto $/ 200 \mathrm{~L}$ de agua, biopreparados a base de $T$. harzianum nativo (C5 y C7) y uno comercial (Natibiol) ambos a dosis de $200 \mathrm{~g}$ del producto a concentración de $2 \times 10^{11}$ UFC $7200 \mathrm{~L}$ de agua, los cuales fueron aplicados a la semilla, al surco al momento de la siembra y al aporque. El diseño usado fue bloques al azar con tres repeticiones. Para los dos ensayos se utilizó la variedad de papa Tibisay resistente a tizón tardío (Phytopthora infestans). Se encontró que $R$. solani grupo GA-3, estuvo presente en los cinco municipios muestreados con incidencias promedio desde $5 \%$ hasta $60 \%$, estando la más alta en Rangel. Bajo condiciones de almacén, la infección estuvo desde 0\% hasta $34 \%$ en $76 \%$ de la semilla informal muestreada. En invernadero los tratamientos a base de Pencycuron y biopreparados tuvieron efectividad similar y a la vez superior a los demás, reduciéndose la infección en plantas y tubérculos. En campo los tratamientos que resultaron superiores fueron los biopreparados a base de aislamientos nativos, estos representan una nueva alternativa para el control de rhizoctoniasis en papa en el Estado Mérida de Venezuela.
\end{abstract}

Palabras claves adicionales: manejo integrado de enfermedades, semilla informal, Rhizoctonia solani, Trichoderma harzianum, biopreparados 


\section{Distribution, Incidence and Alternative of Control in Potato Crop in Mérida, Venezuela}

\section{Summary}

To determine the incidence, distribution level and search for new alternatives to control potato black scurf (Rhizoctonia solani) in the State of Mérida, a survey was done in five potato growing municipalities and a study of informal storage of potato seed tuber in Pico El Águila coming from seven municipalities was also conducted. The evaluations were done under greenhouse and field conditions. Later on, under field and greenhouse conditions alternatives of control to be integrated in handling the disease were evaluated. In the greenhouse, Carboxin +Thiram, Mancozeb, Pencycuron (in commercial doses) and two biopreparations based on Trichoderma harzianum $(200 \mathrm{~g}$ of the product at a concentration of $2 \times 10^{11}$ UFC / $200 \mathrm{~L}$ of water) were applied to seed tubers and soil, and tested against the effect of the disease in an aleatory factorial block design, with three replicates. Meanwhile, in the Mucuchies experimental field station (3100 meter on the sea level), the following treatment were valuated: Pencycuron $(600 \mathrm{~g}$ of the product at a concentration of $2 \times 10^{11}$ UFC $7200 \mathrm{~L}$ of water), two biopreparations of native $T$. harzianum (C5 and $\mathrm{C} 7$ ), and the commercial biopreparation (Natibiol), both in doses of $200 \mathrm{~g}$ of the product at a concentration of $2 \times 10$ UFC $7200 \mathrm{~L}$ of water, were applied to the seed and furrow at planting and hilling. An aleatory Block design with 3 replicates was used. The Tibisay variety, which is resistant to Phytopthora infestans was used also. It was found that a $R$. solani GA-3 group was present in the five sampled municipalities, with a mean level of incidence from $5 \%$ to $60 \%$, being the highest in the municipality of Rangel. Under storage condition, the infection in the seed was from $0 \%$ to $34 \%$ in $76 \%$ in the non formal samples of potato seed tuber. The treatments based on Pencycuron and biopreparations showed a similar behavior, being superior according to their effects in reducing the infection of plant and tuber. In the field, the best treatments were the biopreparations of native $T$. harzianum. The T5 and T7 could be a new alternative to control black scurf in potato crop in Mérida, Venezuela.

Additional Index words: Integrated pest management, informal seed, Rhizoctonia solani, Trichoderma harzianum, biopreparations 


\section{Introducción}

La rhizoctoniasis o costra negra de la papa causada por el hongo Rhizoctonia solani Kühn en la fase asexual y Thanatephorus cucumeris (Frank) Donk en la fase sexual, se han venido incrementando en incidencia y distribución en todas las zonas paperas del estado Mérida. A menudo se observan plantaciones con abundantes plantas achaparradas, amarillentas, con tubérculos aéreos (Figura 1), y aspecto blanquecino entre el cuello de la raíz e inicio del tallo (Figura 2). Estas plantas al ser cosechadas producen tubérculos con evidentes deformaciones, altamente manchados por presencia de esclerocios del hongo y con rajaduras siendo muy bajos los rendimientos (Figura 3), experimentando una reducción que llega a 50\%. (García, et al., 1999). La enfermedad se ha encontrado en las variedades comerciales Granóla, Caribay, Andinita, Tibisay, Atzimba, Monserratt, y Diacol Capiro. En esta última, una variedad colombiana, la incidencia es mayor.

El hongo $R$. solani GA-3 es un habitante natural del suelo que causa el cancro de la papa. Como resultado de su colonización saprofítica 0 parasítica, sus propágulos (micelio y esclerocios) sobreviven en el suelo independientemente y en asociación con materia orgánica o en tejidos del hospedante (10). Los propágulos pueden sobrevivir por varios años sirviendo de inóculo primario para sub-siguientes cultivos (9).

En Balcarce (Provincia de Buenos Aires, Argentina), se cuantificó la magnitud de las pérdidas de calidad que potencialmente podría causar $R$. solani en papa, el 35\% de los tubérculos del cultivar Ballenera sufrió rajaduras por la infección con inóculo natural del patógeno en el campo (5).

No hay una medida de control suficientemente efectiva para proteger la papa del cancro por Rhizoctonia. La combinación de medidas de control químico, resistencia genética, métodos culturales y biológicos permitirán aumentar las posibilidades de control. Cardoso y Echandi (2) y Escande y Echandi (5) lograron controlar la pudrición de raíces de fríjol y el cancro del tallo de papa causados por Rhizoctonia en todos los experimentos mediante el uso de especies binucleadas de Rhizoctonia (RBN).

El hongo Trichoderma harzianum es un antagonista natural del suelo, que ha sido probado y usado con indiscutibles éxitos en el control de $R$. solani en otros cultivos bajo diferentes formas de aplicación $(3,7,11,12,13)$. Este hongo representa una alternativa potencial como componente de manejo de R. solani.

Debido al potencial en aumento de pérdidas que representaría el incremento de rhizoctoniasis de la papa dentro del manejo global del cultivo, usando sólo el producto a base del ingrediente activo Pencycuron, el cual es muy costoso y contaminante, se desarrolló esta investigación con el fin de evaluar la distribución e incidencia de la enfermedad, y a su vez la efectividad de control sobre ésta de biopreparados del antagonista $T$. harzianum. 


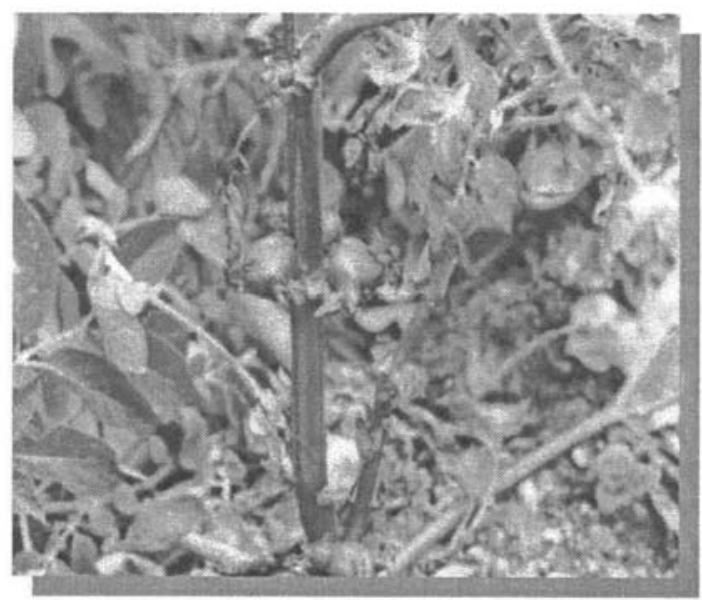

Figura 1. Tubérculos aéreos observados en plantas de papa del cultivar Granola en Mucuchíes, Estado Mérida.

Figura 2. Fase sexual del hongo. Nótese la presencia de puntuaciones blanquecinas en el cuello de plantas de papa cultivar Diacol Capiro.
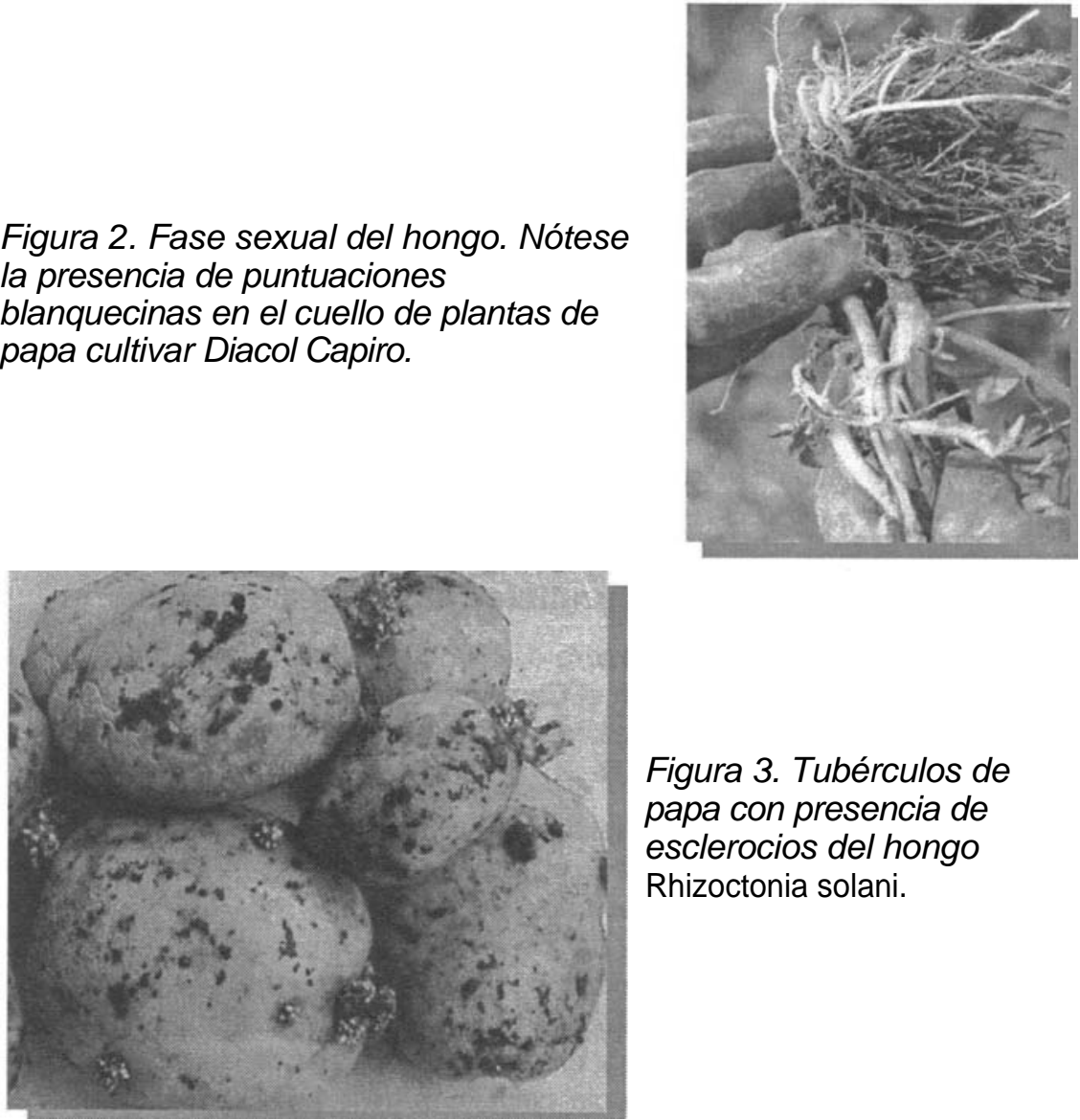

Figura 3. Tubérculos de papa con presencia de esclerocios del hongo Rhizoctonia solani. 


\section{Materiales y Métodos}

\section{Distribución e incidencia de $R$. solani en zonas productoras de papa del estado Mérida}

Se realizó un diagnóstico de campo en los cinco municipios productores de papa más importantes del estado Mérida. Se muestrearon entre $10 \%$ y $20 \%$ de parcelas ubicadas en localidades de los municipios: Libertador, Rangel, Pueblo Llano, Rivas Dávila y Miranda. Se recorrieron, evaluaron y tomaron muestras de tejido en un $20 \%$ de las parcelas sembradas, siguiendo una diagonal en el sembradío y en forma de zigzag a intervalos de 10 plantas. Los criterios de evaluación fueron: distribución de la enfermedad en relación al número de localidades infectadas con $R$. solani, e incidencia de rhizoctoniasis en porcentaje de acuerdo al número de plantas enfermas en relación al número de plantas evaluadas.

\section{Detección de $R$. solani sobre tubérculos de semilla informal}

Se muestrearon 206 huacales de $50 \mathrm{~kg}$ de tubérculos semilla de papa informal, almacenados por los productores en los silos de Pico El Águila, del estado Mérida, ubicado a $4017 \mathrm{msnm}$, bajo una temperatura media diaria de $4^{\circ} \mathrm{C}$. Las muestras se recolectaron en bolsas plásticas, tomando un promedio de 10 tubérculos al azar por cada $50 \mathrm{~kg}$ de semilla almacenada, que luego fueron analizados en el laboratorio. Se tomaron datos sobre número de tubérculos infectados y viabilidad de esclerocios encontrados en la superficie de los mismos. Para ello se sometieron tubérculos infectados a cámara húmeda por 24 horas y luego se sembraron esclerocios en cajas de petri que contenían el medio de cultivo Agar-Papa-Dextrosa. Al obtener cultivos puros se llevaron a la Universidad de los Andes (ULA) para que se determinara el grupo de anastomosis del hongo.

\section{Alternativas de control bajo condiciones de invernadero}

El ensayo fue realizado en un invernadero de techo de vidrio transparente y cubierto de malla antiáfida, ubicado en el Campo Experimental Mucuchíes a una altura de $3100 \mathrm{msnm}$, la temperatura promedio dentro del invernadero fue $18 \pm$ $2^{\circ} \mathrm{C}$ y humedad relativa de $80 \%$.

Entre las alternativas de control se evaluaron dos biopreparados a base de $T$. harzianum, Natibiol comercializado por una empresa privada en Venezuela y un biopreparado de una cepa autóctona del INIA-Mérida, el cual aún no se comercializa, y tres de los fungicidas más comúnmente usados por los productores para desinfección de semilla de papa y/o control en campo, identificados como sigue: $F_{0}=$ Testigo - Sin tratamiento; $F_{1}=$ Carboxin+Thiram (Vitavax) a dosis comercial de $800 \mathrm{mi} / 400 \mathrm{l}$ de agua; $F_{2}=$ Mancozeb (Manzate) a dosis media comercial de $1 \mathrm{~kg} / 400 \mathrm{l}$ de agua; $F_{3}=$ Pencycuron (Monceren) a dosis media comercial de $600 \mathrm{~g} / 400 \mathrm{l}$ de agua; $F_{4}=$ Biopreparado a base del 
antagonista $T$. harzianum (cepa C5) autóctono de la zona a dosis de $200 \mathrm{~g}$ de esporas /200I de agua; $F_{5}=$ Biopreparado a base del antagonista 7. harzianum (cepa C7) autóctono de la zona, a dosis de $200 \mathrm{~g}$ de esporas/2001 de agua, se utilizó $1 \%$ de surfactante. La aplicación de los tratamientos se hizo sobre semilla y suelo (contaminado y sano).

Para evaluar las alternativas anteriores, se sembraron diez (10) tubérculos de la variedad de papa Tibisay por unidad experimental, en bolsas de polietileno que contenían un grupo suelo sano y otro suelo contaminado con el hongo. Cada grupo fue dividido en dos partes, utilizando en una de las partes semilla sana y en la otra contaminada.

El suelo sano correspondió a un sustrato a base de tierra negra, concha de coco y arena $(2: 2: 1)$ esterilizado por 2 horas a $90^{\circ} \mathrm{C}$, y el suelo contaminado provino de una finca donde se observó más de un $90 \%$ de infección con Rhizoctonia en el último sembradío.

El diseño estadístico para los 24 tratamientos dados en la Tabla 1 fue en bloques al azar con arreglo en factorial, con tres repeticiones. Las evaluaciones se realizaron semanalmente tomando datos sobre: número de plantas con síntomas de la enfermedad y número de tubérculos infectados. A los datos obtenidos se les realizó análisis de variancia y prueba de medias de diferencia mínima de significación (LDS).

\section{Alternativas de control bajo condiciones de campo}

La siembra, se realizó en el Campo Experimental Mucuchíes a 3100 msnm, temperatura promedio de $11^{\circ} \mathrm{C}$ y Humedad Relativa Promedio de $75 \%$, en una parcela donde se había presentado un ataque severo de más de $50 \%$ de $R$. solani en papa. Los tratamientos usados fueron: Pencycuron a dosis de $600 \mathrm{~g} / 200$ l de agua; Biopreparados a base de $T$. harzianum autóctono (C5 y C7), a dosis de $200 \mathrm{~g}$ de esporas/2001 de agua; Biopreparado a base de $T$. harzianum comercial (Natibiol), $200 \mathrm{~g}$ de esporas por 2001 de agua. Estos se identificaron como sigue:

$\mathrm{F}_{0}=$ Testigo (Sin aplicación); $\mathrm{F}_{1}=$ Pencycuron; $\mathrm{F}_{2}=$ Biopreparado autóctono (cepa $\left.C_{5}\right) ; F_{3}=$ Biopreparado autóctono $\left(\right.$ cepa $\left.C_{7}\right) ; F_{4}=$ Biopreparado Comercial (Natibiol).

Además, se evaluó como otro parámetro la sanidad de la semilla (sana, infectada).

Las aplicaciones se realizaron a la semilla, sumergiéndola por 30 min (dejando secar por 2 horas), al surco al momento de la siembra y al surco en el aporque.

El diseño estadístico para los 10 tratamientos dados en la Tabla 2 fue bloques al azar con tres repeticiones. Se utilizó la variedad de papa Tibisay, sembrando 4 surcos por tratamiento y 20 plantas/surco. 
Tabla 1. Tratamientos que combinan las alternativas de control con semilla sana o semilla infectada, sembrada en suelo sano o contaminado.

\begin{tabular}{cl}
\hline Tratamientos & \\
\hline & Combinaciones \\
$\mathrm{T}_{0}$ & Suelo sano $\times$ Semilla sana $\times$ Sin tratar. \\
$\mathrm{T}_{1}$ & Suelo sano $\times$ Semilla sana $\times$ Tratada con Carboxin + Thiram. \\
$\mathrm{T}_{2}$ & Suelo sano $\times$ Semilla sana $\times$ Tratada con Mancozeb. \\
$\mathrm{T}_{3}$ & Suelo sano $\times$ Semilla sana $\times$ Tratada con Pencycuron. \\
$\mathrm{T}_{4}$ & Suelo sano $\times$ Semilla sana $\times$ Tratada con biopreparado C5. \\
$\mathrm{T}_{5}$ & Suelo sano $\times$ Semilla sana $\times$ Tratada con biopreparado C7. \\
$\mathrm{T}_{6}$ & Suelo sano $\times$ Semilla infectada $\times$ Sin tratar. \\
$\mathrm{T}_{7}$ & Suelo sano $\times$ Semilla infectada $\times$ Tratada con Carboxin + Tirad \\
$\mathrm{T}_{8}$ & Suelo sano $\times$ Semilla infectada $\times$ Tratada con Mancozeb. \\
$\mathrm{T}_{9}$ & Suelo sano $\times$ Semilla infectada $\times$ Tratada con Pencycuron. \\
$\mathrm{T}_{10}$ & Suelo sano $\times$ Semilla infectada $\times$ Tratada con biopreparado \\
& C5. \\
$\mathrm{T}_{11}$ & Suelo sano $\times$ Semilla infectada $\times$ Tratada con biopreparado \\
& C7. \\
$\mathrm{T}_{12}$ & Suelo infectado $\times$ Semilla sana $\times$ Sin tratar. \\
$\mathrm{T}_{13}$ & Suelo infectado $\times$ Semilla sana $\times$ Tratada con Carboxin + \\
$\mathrm{T}_{14}$ & Thiram. \\
$\mathrm{T}_{15}$ & Suelo infectado $\times$ Semilla sana $\times$ Tratada con Mancozeb. \\
$\mathrm{T}_{16}$ & Suelo infectado $\times$ Semilla sana $\times$ Tratada con Pencycuron \\
$\mathrm{T}_{17}$ & Suelo infectado $\times$ Semilla sana $\times$ Tratada con biopreparado \\
& C5. \\
$\mathrm{T}_{18}$ & Suelo infectado $\times$ Semilla sana $\times$ Tratada con biopreparado \\
$\mathrm{T}_{19}$ & Suelo infectado $\times$ Semilla infectada $\times$ Sin tratar. \\
$\mathrm{T}_{20}$ & Suelo infectado $\times$ Semilla infectada $\times$ Tratada con Carboxin + \\
$\mathrm{T}_{21}$ & Tirad \\
$\mathrm{T}_{22}$ & Suelo infectado $\times$ Semilla infectada $\times$ Tratada con Mancozeb. \\
$\mathrm{T}_{23}$ & Suelo infectado $\times$ Semilla infectada $\times$ Tratada con Pencycuron. \\
& biopreparado C5. \\
& Suelo infectado $\times$ Semilla infectada $\times$ Tratada con \\
&
\end{tabular}


Tabla 2. Tratamientos que combinan las alternativas de control con semilla sana o semilla infectada, bajo condiciones de campo.

Tratamientos Combinaciones

\begin{tabular}{ll}
\hline $\mathrm{T}_{0}$ & Semilla sana sin tratar. \\
$\mathrm{T}_{2}$ & Semilla sana tratada con Pencycuron \\
$\mathrm{T}_{3}$ & Semilla sana tratada con biopreparado $\mathrm{C} 5$. \\
$\mathrm{T}_{4}$ & Semilla sana tratada con biopreparado $\mathrm{C} 7$. \\
$\mathrm{T}_{5}$ & Semilla sana tratada con biopreparado Natibiol \\
$\mathrm{T}_{6}$ & Semilla infectada sin tratar. \\
$\mathrm{T}_{7}$ & Semilla infectada tratada con Pencycuron. \\
$\mathrm{T}_{8}$ & Semilla infectada tratada con biopreparado $\mathrm{C} 5$. \\
$\mathrm{T}_{9}$ & Semilla infectada tratada con biopreparado C7. \\
\hline
\end{tabular}

Para asegurar la colonización del hongo antagonista se aplicó $50 \mathrm{Kg}$. de fertilizante químico $12-12-17 / 2$ por $100 \mathrm{Kg}$. de semilla de papa, más $50 \mathrm{Kg}$. de cachaza de caña de azúcar y $50 \mathrm{Kg}$. de humus sólido por $50 \mathrm{~kg}$ de semilla de papa, de acuerdo al análisis de suelo.

Las evaluaciones se
realizaron semanalmente
sobre los dos surcos
centrales, tomando datos de:
número de plantas enfermas
con síntomas y grado de
infección en los tubérculos
(Figura 4). Se utilizó una
escala de infección de
tubérculos de O a 3, donde
cero significa tubérculo
totalmente sano, libre de
esclerocios, y 3 tubérculos
infectados con abundantes
esclerocios que cubren más
del $20 \%$ de la piel (Tabla 3).
También se tomaron datos de
número de tubérculos in-
fectados, rendimiento y tu-
bérculos de descarte, en kg.

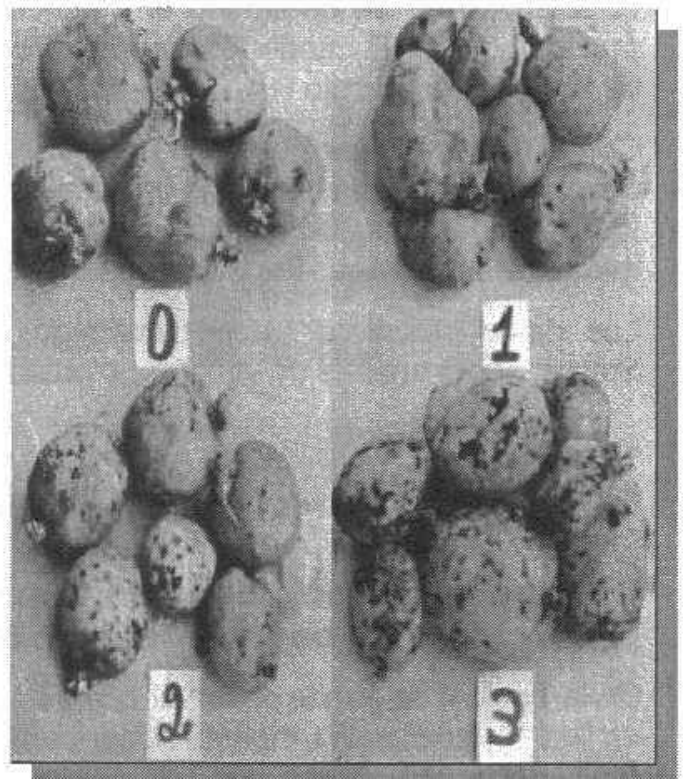

Figura 4. Diferentes grados de infectación de tubérculos de papa ocasionados por

R. Solani. 
Tabla 3. Escala de daño ocasionado por Rhizoctonia solani en tubérculos de papa (severidad)

\begin{tabular}{lcl}
\hline Grado & Cualidad & Daños Causados \\
\hline 0 & Sana & $\begin{array}{l}\text { Tubérculos totalmente sanos libres de } \\
\text { esclerocios o lesiones ausentes. }\end{array}$ \\
\hline 1 & Baja incidencia & Tubérculos con esclerocios de \\
(B) & $\begin{array}{l}\text { pequeños a medianos en número de } \\
\text { entre 1 y 5, cubriendo hasta el 10\% de } \\
\text { la piel, ó lesiones < 5 mm de longitud. }\end{array}$ \\
\hline 2 & Mediana incidencia & Tubérculos con esclerocios medianos \\
& (M) & $\begin{array}{l}\text { en número de entre 6 y 10, cubriendo } \\
\text { entre 10 y 20\% de la piel, ó lesiones }>\end{array}$ \\
& 5 mm de longitud. \\
\hline 3 & Alta incidencia & Tubérculos con grandes esclerocios \\
& (A) & prominentes, en número mayor de 10, \\
& cubriendo más del 20\% de la piel, con \\
& piel enmallada altamente deformados \\
& o lesiones que rodean al tallo. \\
\hline
\end{tabular}

La cobertura de los tubérculos en red o con esclerocios causados por $R$. solani GA-3, se determinó en tubérculos lavados y mojados; según la metodología de Escande (6), con modificaciones adaptadas a la expresión de la enfermedad bajo las condiciones de Mérida.

Con el fin de verificar la recolonización del antagonista al suelo, al finalizar la cosecha se tomaron muestras de tierra de parcelas que fueron tratadas con los biopreparados. Se realizaron cultivos en placas petri que contenían el medio de cultivo Agar-Papa-Dextrosa.

Los datos obtenidos fueron analizados en el programa computarizado Statisti, sobre análisis de variancia y pruebas de medias de LDS

\section{Resultados y Discusión}

\section{Distribución e incidencia de $\boldsymbol{R}$. solani en zonas productoras de papa del estado Mérida}

En la Tabla 4 se puede observar que $R$. solani GA-3 se encuentra presente en las 17 localidades muestreadas, pertenecientes a los cinco municipios productores, en alturas que van desde 1800 hasta $3500 \mathrm{msnm}$, con una incidencia promedio que varía entre 5\% y $60 \%$, ubicándose los valores de incidencia más altos en Rangel donde más del $70 \%$ de las localidades presentó incidencia superior al 30\%. 
Tabla 4. Distribución e incidencia de $R$. solani en zonas productoras de papa del estado Mérida.

\begin{tabular}{lll|c|c}
\hline $\begin{array}{c}\text { Distribución/ } \\
\text { Municipio }\end{array}$ & Localidad & $\begin{array}{c}\text { Altura } \\
\text { msnm }\end{array}$ & $\begin{array}{c}\text { Número de } \\
\text { parcelas } \\
\text { por } \\
\text { localidad }\end{array}$ & $\begin{array}{c}\text { Rango de } \\
\text { incidencia } \\
\text { (\%) }\end{array}$ \\
\hline \multirow{3}{*}{ Libertador } & La Caña & 2.100 & 3 & 5 \\
& La Vega & 2.200 & 2 & $26-50$ \\
& Bella Vista & 1.800 & 3 & $10-25$ \\
& La Bravera & 2.000 & 3 & $10-20$ \\
\hline \multirow{4}{*}{ Rangel } & Llano El Hato & 3.500 & 4 & $5-30$ \\
& Misintá & 3.200 & 4 & 30 \\
& La Toma & 3.000 & 3 & $10-60$ \\
& Mucumpate & 3.200 & 4 & 50 \\
& Mucubají & 4.000 & 10 & 55 \\
Pueblo Llano & Apartaderos & 3.500 & 3 & 45 \\
& Miyoy & 1.800 & 3 & 5 \\
& El Helechal & 2.200 & 3 & $5-10$ \\
& La Culata & 2.900 & 3 & 10 \\
\multirow{2}{*}{ Rivas Dávila } & El Arbolito & 2.500 & 4 & 25 \\
& La M & 2.500 & 4 & 30 \\
& Las Playitas & 2.200 & 3 & 20 \\
& Bailadores & 2.000 & 3 & 5 \\
\hline
\end{tabular}

Lo anterior evidencia que $R$. solani se ha convertido en un serio problema para la producción papera del Estado Mérida.

Resultados similares fueron encontrados por Calderón y FernándezNorthcote (1), quienes señalaron que la rhizoctoniasis denominada también "cancro del tallo", "costra negra", es una enfermedad ampliamente diseminada en las zonas paperas de Solivia, alcanzando incidencias hasta del $90 \%$ en los departamentos de Cochabamba, Chuquisaca, Potosí, Oruro, La Paz y Tarija. Las plantas severamente afectadas con rhizoctoniasis muestran una disminución en rendimiento hasta del $96 \%$. Asimismo, señalan que el efecto en la calidad es altamente significativo en relación al de las plantas sanas, pudiendo ocasionar cosechas con $80 \%$ de tubérculos pequeños y deformes y sólo un $20 \%$ de tamaño regular.

\section{Detección de $\boldsymbol{R}$. solani sobre tubérculos semilla informal}

En la Tabla 5 se puede observar los niveles de infectación de la semilla proveniente de 25 localidades muestreadas. De éstas, 19 presentaron la enfermedad y sólo seis estuvieron libres de ella. Esto representa un 76\% de localidades con semilla infectada por rhizoctoniasis bajo un promedio general de $41 \%$. Mientras que la incidencia estuvo entre 0 y $34 \%$ con un promedio de $10 \%$. 
Tabla 5. Detección de Rhizoctonia solani en tubérculos semillas informal proveniente de 25 localidades del Estado Mérida y almacenados en silos del Pico El Águila. 1997-1998.

\begin{tabular}{|c|c|c|c|c|c|c|}
\hline Municipio & Localidad & $\begin{array}{l}\text { No. de } \\
\text { guacal }\end{array}$ & $\begin{array}{c}\text { No. de } \\
\text { muestras }\end{array}$ & $\begin{array}{c}\text { No. de } \\
\text { tubérculos } \\
\text { analizados }\end{array}$ & $\begin{array}{c}\text { Distribución } \\
(\%)\end{array}$ & $\begin{array}{c}\text { Incidencia } \\
\text { en } \\
\text { tubérculos } \\
(\%)\end{array}$ \\
\hline \multirow[t]{13}{*}{ Rangel } & Mucuchíes & 542 & 14 & 140 & 86 & 16 \\
\hline & Apartaderos & 130 & 1 & 10 & 100 & 20 \\
\hline & Mucumpate & 424 & 8 & 80 & 63 & 15 \\
\hline & Escaguey & 245 & 4 & 40 & 25 & 25 \\
\hline & Mucurubá & 442 & 5 & 50 & 100 & 34 \\
\hline & $\begin{array}{l}\text { Llano El } \\
\text { Hato }\end{array}$ & 200 & 1 & 10 & 100 & 20 \\
\hline & Misintá & 77 & 3 & 30 & 66 & 20 \\
\hline & La Toma & 76 & 2 & 20 & 0 & 5 \\
\hline & Tropicon & 70 & 1 & 10 & 0 & 0 \\
\hline & $\begin{array}{l}\text { Mesa } \\
\text { Grande }\end{array}$ & 32 & 1 & 10 & 0 & 0 \\
\hline & Mucuchache & 88 & 2 & 20 & 100 & 30 \\
\hline & Mucuy & 160 & 2 & 20 & 0 & 0 \\
\hline & gaviria & 12 & 1 & 10 & 100 & 10 \\
\hline Promedio & & & & & 57 & 15 \\
\hline \multirow[t]{3}{*}{ Miranda } & Timotes & 169 & 6 & 60 & 50 & 5 \\
\hline & La Venta & 25 & 1 & 10 & 100 & 10 \\
\hline & $\begin{array}{l}\text { Cruz } \\
\text { Chiquita }\end{array}$ & 72 & 1 & 10 & 100 & 20 \\
\hline Promedio & & & & & 83 & 12 \\
\hline Pueblo Llano & Pueblo Llano & 1670 & 44 & 440 & 54 & 11 \\
\hline \multirow[t]{4}{*}{ C. Quintero } & S. Domingo & 898 & 27 & 270 & 66 & 15 \\
\hline & Efafiche & 58 & 2 & 20 & 100 & 15 \\
\hline & Aracay & 212 & 7 & 70 & 42 & 7 \\
\hline & Las Piedras & 31 & 3 & 30 & 0 & 0 \\
\hline Promedio & & & & & 52 & 9 \\
\hline S. Marquina & Tabay & 140 & 4 & 40 & 0 & 0 \\
\hline Libertador & $\begin{array}{l}\text { El Valle } \\
\text { El Morro }\end{array}$ & $\begin{array}{l}43 \\
98\end{array}$ & $\begin{array}{l}1 \\
3\end{array}$ & $\begin{array}{l}10 \\
30\end{array}$ & $\begin{array}{c}0 \\
66\end{array}$ & $\begin{array}{c}0 \\
16\end{array}$ \\
\hline Promedio & & & & & 33 & 8 \\
\hline C. Elias & Jají & 42 & 2 & 20 & 50 & 15 \\
\hline $\begin{array}{l}\text { Gran } \\
\text { Promedio }\end{array}$ & & & & & 41 & 10 \\
\hline
\end{tabular}

Lo anterior evidencia la importancia que tiene la semilla como fuente de inóculo y de diseminación de la enfermedad en el estado Mérida, y explica la alta distribución e incremento de rhizoctoniasis.

\section{Alternativas de control bajo condiciones de invernadero}

El análisis de variancia del porcentaje de plantas y tubérculos infectados con rhizoctoniasis, mostró diferencias estadísticamente significativas entre los tratamientos. Esto quiere decir que al menos uno de los tratamientos es diferente de los demás. 
La Tabla 6 señala los resultados de la efectividad de los tratamientos sobre $R$. solani, donde todos los tratamientos se ubicaron al mismo nivel de los testigos sanos. El Pencycuron y los biopreparados autóctonos tuvieron un comportamiento similar y más efectivo que los demás en términos numéricos, logrando reducir el porcentaje de tubérculos infectados hasta 0\%, desde un 33.33\% observado en el testigo enfermo (SuC x SemC x FO). Siguiendo un orden de efectividad de los tratamientos, los mejores fueron: aplicación del biopreparado C5 sobre suelo contaminado y semilla sana (SuC x SemS x F4, SuC x SemC) con 0\% de tubérculos infectados, aplicación de Pencycuron sobre suelo contaminado y semilla sana (SuC $x$ SemS x F3) y sobre suelo sano y semilla infectada (SuS x SemC x F3) con $2.23 \%$ y $3.87 \%$; tratamiento con biopreparado C7 sobre suelo sano y semilla contaminada ( SuS x SemC x F5) y sobre suelo contaminado y semilla infectada (SuC x SemC x F5) con $2.47 \%$ y $3 \%$ respectivamente.

Tabla 6. Efectividad del uso de tres fungicidas y dos biopreparados en el control de la rhizoctoniasis bajo condiciones de invernadero. Mucuchies-Mérida. 1999.

\begin{tabular}{|c|c|c|}
\hline Tratamientos & $\begin{array}{l}\text { Plantas infectadas } \\
\text { (\%) }\end{array}$ & $\begin{array}{c}\text { Tubérculos infectados } \\
\text { (\%) }\end{array}$ \\
\hline SuC $\times$ SemS x F2 (T20) & $46,67 \mathrm{a}$ & 29.17 abcd \\
\hline SuSxSemxF4(T10) & $40,00 a b$ & 23.60 bcde \\
\hline SuCxSemSxF2(T14) & $33,33 a b c$ & 9.47 def \\
\hline SuCxSemCxFO(T18) & $33,33 a b c$ & 20.67 bcde \\
\hline SuCxSemSxF5(T17) & $30,00 \mathrm{bcd}$ & 22.30 bcde \\
\hline SuS x SemC x FO (T6) & $23,33 \mathrm{bcd}$ & 22.37 bcde \\
\hline SuS x SemC x F1 (T7) & $23,33 \mathrm{bcd}$ & $32.40 a b c$ \\
\hline SuC x SemS xF1 (T13) & $23,33 \mathrm{bcd}$ & $33.40 a b$ \\
\hline SuC $\times$ SemC xF1 (T19) & $23,33 \mathrm{bcd}$ & 18.00 bcdef \\
\hline SuS x SemC xF5(T11) & 20,00 bcde & 2.47 ef \\
\hline SuCcSemxFO(T12) & 20,00 bcde & 5.40 bcdef \\
\hline SuS x SemC x F2 (T8) & 20,00 bcde & $49.10 \mathrm{a}$ \\
\hline SuC x SemC xF3(T21) & 16,67 cde & 12.83 bcdef \\
\hline SuC $\times$ SemC $\times$ F5 (T23) & 13,33 cde & 3.00 ef \\
\hline SuCxSemSxF3(T15) & 6,67 de & 2.23 ef \\
\hline SuCxSemSxF4(T16) & 6,67 de & $0 \mathrm{f}$ \\
\hline SuS x SemC x F3 (T9) & 6,67 de & 3.87 ef \\
\hline SuS x SemS x F4 (T4) & $0 \mathrm{e}$ & $0 \mathrm{f}$ \\
\hline SuS x SemS x F3 (T3) & $0 \mathrm{e}$ & $0 \mathrm{f}$ \\
\hline SuS x SemS x F2 (T2) & $0 \mathrm{e}$ & $0 \mathrm{f}$ \\
\hline SuS x SemS x FO (TO) & $0 \mathrm{e}$ & $0 \mathrm{f}$ \\
\hline SuS x SemS xF1 (T1) & $0 \mathrm{e}$ & $0 \mathrm{f}$ \\
\hline SuC $x$ SemC x F4 (T22) & $0 \mathrm{e}$ & $0 \mathrm{f}$ \\
\hline SuS x SemS x F5 (T5) & $0 \mathrm{e}$ & $0 \mathrm{f}$ \\
\hline
\end{tabular}

SuS = Suelo Sano; SemS = Semilla Sana; SuC = Suelo Contaminado; SemC Semilla contaminada. FO = Testigo, sin tratamiento; F1 = Carboxin + Tiram; F2 Mancozeb; F3 = Pencycuron; F4 = Biopreparado (C5); F5 = Biopreparado (C7). 
Los promedios de cada columna que tienen una letra en común no difieren entre sí, según la prueba de LDS $(P<0,05)$. En cuanto a porcentaje de plantas enfermas, el tratamiento a base de Carboxin + Thiram (SuS $x$ SemC x F1, SuC x SemS x F1, SuC x SemC x F1) tuvo un comportamiento igual que los testigos enfermos (SuC x SemC x FO, SuS x SemC x FO, SuCx SemS x FO). Mientras que los tratamientos que tuvieron un mejor comportamiento fueron: aplicación de biopreparado C5 (SuC x SemC x F4, SuS x SemS x F4) logrando disminuir este porcentaje entre 0\% y $6.67 \%$ respectivamente; aplicación de Pencycuron (SuC x SemS x F3, SuS x SemC x F3) con $6.67 \%$ y la aplicación del biopreparado C7 (SuC x SemC x F5) con $13.33 \%$.

Estos resultados muestran la potencialidad del uso de los aislamientos autóctonos del hongo antagonista $T$. harzianum para el control de $R$. solani.

\section{Alternativas de control bajo condiciones de campo}

El análisis de variancia reportó diferencias significativas entre los tratamientos para todas las variables evaluadas.

La Tabla 7 muestra que todos los tratamientos tuvieron un buen comportamiento en relación a los testigos (siembra de semilla sana y siembra de semilla contaminada sin tratar), formaron un grupo aparte en relación a los demás. Mientras que para porcentaje de plantas infectadas tuvo mejor comportamiento el biopreparado C7 con $0 \%$ de plantas sintomáticas; aunque no existen diferencias estadísticas significativas. Este mismo tratamiento, en cuanto a porcentaje de tubérculos infectados, logró mantener valores bajos de infectación entre $26.40 \%$ y $31.47 \%$ cuando se sembró semilla sana y contaminada, respectivamente.

En cuanto al porcentaje de tubérculos descartados se observa que los tratamientos que mostraron mejor comportamiento fueron los biopreparados, tanto el autóctono como el introducido. Estando este en un mínimo de 5.17\% para cuando se aplicó biopreparado C5 y se usó semilla contaminada (SemC x F2) en relación a los testigos los cuales estuvieron en $10.12 \%$ (SemC x FO) y 17.28\% (SemS x FO) usando semilla contaminada y sana y ubicándose en el mismo grupo.

En cuanto a rendimiento, las parcelas tratadas con los biopreparados C5 Y $\mathrm{C} 7$, mostraron un mejor comportamiento siendo superior el tratamiento a base de biopreparado C5 con 44,590 Kg./ha estimado y 40,078 Kg./ha, cuando se sembró semilla contaminada y sana. Esto seguido del tratamiento a base del biopreparado $\mathrm{C} 7$ con 38,255 Kg./ha y 34,336 Kg./ha cuando se sembró semilla contaminada y sana respectivamente.

En la Figura 5, se muestran los niveles de intensidad de daño en tubérculos infectados por $R$. solani. Se observa que con los tratamientos a base de Pencycuron y biopreparado, los tubérculos afectados presentaron niveles bajos de intensidad de daño. En estos tratamientos, sólo un menor porcentaje de tubérculos tuvo daños en alto grado, estando los menores 
valores en el tratamiento a base del biopreparado C7 con $0.80 \%$ y $1.62 \%$, en relación a los testigos, en los cuales se encontró entre $7.42 \%$ y $9.80 \%$ de tubérculos con un alto nivel de infectación, para cuando se uso semilla infectada y sana respectivamente.

Tabla 7. Efectividad del uso de un funguicida y tres biopreparados en el control de rhizoctoniasis de la papa bajo condiciones de campo. Mucuchíes Mérida 1999.

\begin{tabular}{|c|c|c|c|c|c|}
\hline \multicolumn{2}{|c|}{ Tratamiento } & \multirow{2}{*}{$\begin{array}{c}\begin{array}{c}\text { Plantas } \\
\text { Infectadas } \\
(\%)\end{array} \\
27.22 \mathrm{a}\end{array}$} & \multirow{2}{*}{$\begin{array}{c}\begin{array}{c}\text { Tubérculos } \\
\text { Infectados } \\
(\%)\end{array} \\
52.38 \mathrm{a}\end{array}$} & \multirow{2}{*}{ 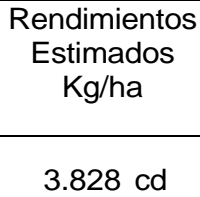 } & \multirow{2}{*}{$\begin{array}{c}\begin{array}{c}\text { Descarte } \\
(\%)\end{array} \\
10.12 \mathrm{ab}\end{array}$} \\
\hline SemC XFO & (T5) & & & & \\
\hline SemS $\times$ FO & (TO) & $27.11 \mathrm{a}$ & $55.10 \mathrm{ab}$ & $14.479 \mathrm{~d}$ & $17.28 \mathrm{a}$ \\
\hline SemS $\times$ F1 & (T1) & $11.11 \mathrm{~b}$ & $26.72 \mathrm{~cd}$ & $19.833 \mathrm{~d}$ & $9.69 a b$ \\
\hline SemC $\times F 1$ & (T6) & $10.00 \mathrm{bc}$ & $20.13 \mathrm{abcd}$ & $32.619 \mathrm{bcd}$ & $8.06 a b$ \\
\hline SemS $\times$ F4 & (T4) & $9.30 \mathrm{bc}$ & $41.03 \mathrm{abcd}$ & $23.184 \mathrm{~cd}$ & $10.33 a b$ \\
\hline SemC $\times$ F4 & (T9) & $9.28 \mathrm{bc}$ & $53.48 \mathrm{ab}$ & $29.987 \mathrm{~d}$ & 7.33 b \\
\hline SemS $\times F 2$ & (T2) & $6.67 \mathrm{bc}$ & $33.09 \mathrm{bcd}$ & $40.078 \mathrm{ab}$ & $5.97 \mathrm{~b}$ \\
\hline SemC $\times F 2$ & (T7) & $2.22 \mathrm{c}$ & $52.64 \mathrm{ab}$ & $44.590 \mathrm{a}$ & $5.17 b$ \\
\hline SemC $\times$ F3 & (T8) & $1.11 \mathrm{bc}$ & $31.47 \mathrm{bcd}$ & $38.255 \mathrm{abc}$ & $6.18 b$ \\
\hline SemS $\times$ F3 & (T3) & $0 \mathrm{c}$ & $26.40 \mathrm{~d}$ & 34.336 bc & $6.71 \mathrm{~b}$ \\
\hline
\end{tabular}

SemC = Semilla Contaminada; SemS = Semilla Sana; FO = Testigo, sin tratamiento; F1 = Pencycuron; F2 = Biopreparado C5; F3 = Biopreparado C7; $\mathrm{F} 4=$ Natibiol.

Los promedios de cada columna que tienen una letra en común no difieren entre sí, según la prueba de LDS $(P<0,05)$.

El hongo $T$. harzianum tuvo mejor actuación cuando se aplicó sobre semilla contaminada, esto se explica porque los esclerocios de Rhizoctonia le sirvieron de alimento para sobrevivir y multiplicarse durante el desarrollo del ciclo de la planta.

Los resultados permiten inferir que el antagonista $T$. harzianum ejerce un buen control de $R$. solani bajo condiciones de campo, protegiendo al cultivo de la enfermedad. Este antagonista puede ser usado como un componente alternativo de biocontrol dentro del manejo integrado de la rhizoctoniasis de la papa. 


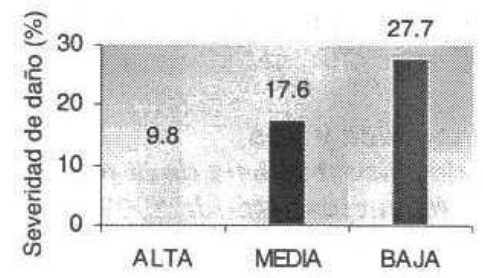

A. Sin tratar

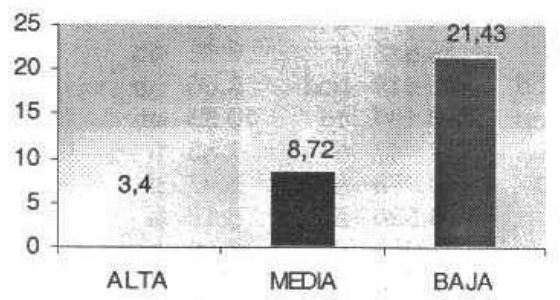

C. Tratamiento con $\mathrm{C5}$

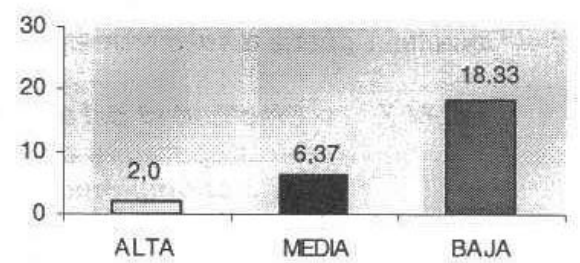

B. Tratamiento con Pencycuron

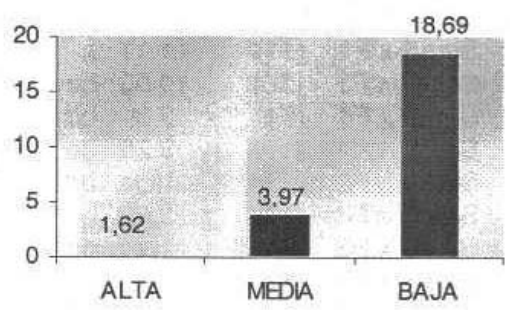

D. Tratamiento con $\mathrm{C7}$

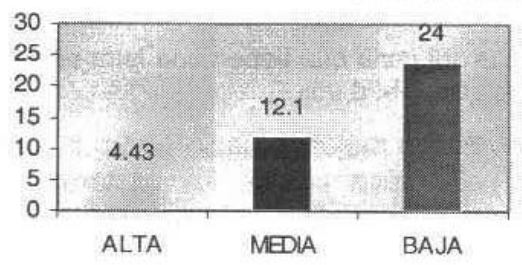

E. Tratamiento con Natibiol

Figura 5. Niveles de severidad de daño ocasionado por Rhizoctonia solani en tubérculos. 


\section{Conclusiones}

- Rhizoctonia solani representa un factor limitante importante en la producción de tubérculos de papa en el estado Mérida en Venezuela, debido a que se ha venido distribuyendo en todas las zonas productoras de importancia, encontrándose incidencias desde 5 hasta 60\%; siendo el municipio Rangel el más altamente afectado.

- La semilla informal de papa producida en la zona es una fuente importante de diseminación de la enfermedad debido a que el $76 \%$ de ésta posee considerables niveles de infección, desde 0 hasta $34 \%$.

- Los biopreparados a base de aislamientos autóctonos de T. harzianum representan una alternativa eficiente $y$ menos contaminante para el control de la rhizoctoniasis de la papa.

\section{Referencias Bibliográficas}

1. Calderón, C., E.N. Fernández Northcote. 1996. Como reconocer y evitar la Rizoctoniasis en el cultivo de la papa. Instituto Boliviano de Tecnología Agropecuaria IBTA. Programa de Investigación de la Papa (PROINPA). Fitopatología Ficha Técnica No. 6.

2. Cardoso, J., E. Echandi. 1987. Biological control of Rhizoctonia root rol of snap bean with binucleate Rhizoctonia-like fungi. Plant Dis. 71:167-170.

3. Elad, Y., Y. Hadar, E. Hadar, I. Chet, Y. Henis. 1981. Biological control of Rhizoctonia solani by Trichoderma harzianum in carnation. Plant Disease. 65:675-677.

4. Escande, A. 1991. Eficacia de especies binucleadas de Rhizoctonia para el control biológico de enfermedades de papa. Informe del Plan de trabajo de actividades priorizada 03: 0127 INTA, EEA, Balcarce (Argentina). $9 \mathrm{p}$.

5. Escande, A., E, Echandi. 1991. Protection of potato from Rhizoctonia canker with binucleate Rhizoctonia spp. Plant Pathol. 40:197-202.

6. Escande A. 1993. Eficacia de especies de Rhizoctonia y del Sulfato de Calcio para el control del cancro. En: Memorias del Seminario Taller sobre Control Integrado de las Principales Enfermedades Fungosas de la Papa. 4-6 Octubre Bella Vista, Uruguay, pp. 59-64.

7. Harman, G.E., I. Chet, R. Baker. 1980. Trichoderma hamatum effects on seed and seedling disease induced in radish and pea by Pythium spp. and Rhizoctonia solani. Phytopathology. 10: 1167-1172. 
8. García, R., A. García, C. Garnica. 1999. ¡Cuidado con la rizoctoniasis de la papa!. Diario Frontera. Página Agropecuaria 5-C. Mérida 3 de enero.

9. Lewis, J., G. Papavizas. 1991. Biocontrol of cotton damping-off caused by Rhizoctonia solani in the field with formulations of Trichoderma spp. and Gliocladium virens. Crop Protection 10: 396402.

10. Papavizas, G., R. Lumsden. 1980. Biological Control of Soilborne fungal propagules. Ann.Rev. Phytopathol. 18: 389-413.

11. Strashnow, Y., Y. Elad, A. Sivan; I. Chet. 1985. Integrated control of Rhizoctonia solani by metil bromide and Trichoderma harzianum. Plant Pathology. 34: 146-51-

12. Sandoval, LL, M. López, D. García, I. Mendoza. 1995. Trichoderma harzianum (cepa A 34): Un biopreparado de amplio espectro para micopatologías del tomate $y$ del pimiento. Instituto de Investigaciones de Sanidad Vegetal de La Habana, Cuba. Boletín Técnico. No. 3.

13. Windham, M; T, Y. Elad, R. Baker, R. 1986. A mechanism for increase plant growth induced by Trichoderma spp. Phytopathology. 76:518-5 